\title{
ÚNG DỤNG MÔ HÌNH TIN TRONG CÔNG TÁC KIỂM TRA SỐ LIỆU ĐO SÂU HỒI ÂM BẰNG PHƯONG PHÁP ĐƠN TIA
}

\author{
ThS. NGUYẼ̃N CÔNG SƠN ${ }^{(1)}$, TS. TRẦ THÙY DƯƠNG(2), KS. VŨ HÔNG TÂP((3) \\ (1) Viện Khoa học Đo đạc và Bản đồ \\ (2)Trường Đại học Mỏ Địa chất \\ (3)Trung tâm Trắc địa Bản đồ Biển
}

$\mathrm{L}$ ưới tam giác không quy chuẩn Triangular Irrigular Network (TIN) từ lâu được biết đến như một công cụ hữu hiệu trong việc lập mô hình số địa hình bởi tính chất linh hoạt và sự mô phỏng rất tốt của nó với những địa hình bị chia cắt mạnh. Qua nghiên cứu và triển khai thực tế, nhóm tác giả đã phát hiện ra những ứng dụng hết sức hữu ích của mô hình TIN trong việc mô hình hoá và tổ chức tìm kiếm. Bài báo khoa học này giới thiệu một trong những ứng dụng như vậy. Các kết quả nghiên cứu của bài báo này là một cơ sở quan trọng cho việc mở rộng phạm vi ứng dụng của mô hình TIN.

\section{1. Đặt vấn đề}

Ở nước ta hiện nay, phương pháp đo sâu hồi âm đơn tia là phương pháp phổ biến để đo vẽ thành lập bản đồ địa hình đáy biển. Số liệu đo sâu thu được là rất quan trọng, vì vậy để đánh giá được độ chính xác kết quả đo sâu thì sau khi đo xong các tuyến đo chính sẽ tiến hành đo các tuyến đo kiểm tra. Việc kiểm tra được thực hiện dựa vào điểm giao cắt giữa đường kiểm tra và đường đo sâu [4], tại vị trí giao cắt chúng ta xác định được tọa độ $(X, Y)$ và 2 giá trị độ sâu, đó là: độ sâu được nội suy từ 2 điểm đo sâu gần nhất trước và sau giao điểm trên từng tuyến đo sâu ( $D_{\text {đstisuy }}^{\text {noi }}$ ); và độ sâu được nội suy từ 2 điểm kiêm tra gần nhất trước và sau giao điểm trên từng tuyến kiểm tra ( $\left.D_{k t}^{\text {nôisuy }}\right)$. Hai giá trị độ sâu nội suy này chính là cơ sở để so sánh, đánh giá kết quả đo sâu. Do dư liệu đo sâu địa hình đáy biển là rất lớn từ hàng trăm nghìn đến vài triệu điểm nên công tác kiểm tra mất rất nhiều thời gian; vì vậy việc tìm hiểu, nghiên cứu phương pháp để kiểm tra, đánh giá được số liệu đo sâu hồi âm một cách nhanh chóng và cho độ chính xác cao là cần thiết. Bài báo này sẽ đưa ra kết quả của việc nghiên cứu và ứng dụng mô hình TIN để giải quyết vấn đề trên.

\section{Giải quyết vấn đề}

\subsection{Phương pháp đo sâu hồi âm đơn tia}

Phương pháp đo sâu hồi âm đơn tia SBES (Single Beam EchoSounder) là phương pháp xác định độ sâu dựa trên cơ sở xác định thời gian lan truyền sóng âm thanh phát đi từ đầu biến âm (Transducer) trong môi trường nước sau khi gặp đáy biển sẽ phản hồi lại đầu biến âm, (hình 1).

Khi thời gian và tốc độ truyền sóng âm trong cột nước được biết thì độ sâu được tính bằng công thức sau:

$$
D=\frac{1}{2} \cdot v \cdot \tau
$$

trong đó: $v$ - Vận tốc truyền sóng âm trong cột nước; $\mathrm{T}$ - Thời gian truyền tín hiệu của sóng âm từ lúc phát tín hiệu tại đầu biến âm xuống đáy biển và quay trở lại; $D$ - Độ sâu điểm đo.

\subsection{Công tác đo sâu hồi âm đơn tia}

Công tác đo đạc, thành lập bản đồ địa 
hình đáy biển tỷ lệ 1:50.000 bằng hệ thống đo sâu hồi âm đơn tia được thực hiện theo quy định [4]. Trong đó công tác đo sâu và việc Fix số liệu phải đảm bảo các yêu cầu sau:

- Khoảng cách giữa 2 tuyến đo sâu liên tiếp là $500 \mathrm{~m}$;

- Các tuyến được đo song song với nhau và theo chiều dốc của địa hình;

- Tọa độ $(X, Y, D)$ của hai điểm liên tiếp trên một tuyến đo sâu được xác định (Fix) với khoảng cách nhỏ hơn $100 \mathrm{~m}$; Tuyến đo sâu được thiết kế như hình 2.
2.3. Công tác kiểm tra số liệu đo sâu hồi âm đơn tia

Số liệu đo sâu là rất quan trọng vì vậy công tác kiểm tra được thực hiện trong suốt quá trình thi công. Để đánh giá được kết quả đo sâu thì sau khi đo xong các tuyến đo chính sẽ tiến hành đo các tuyến đo kiểm tra, các tuyến kiểm tra được đo theo hướng vuông góc với tuyến đo chính. Các tuyến kiểm tra cũng tuân thủ theo quy định kỹ thuật thành lập bản đồ địa hình đáy biển (theo tỷ lệ tương ứng). Đối với bản đồ 1/50.000 khoảng cách giữa hai tuyến đo

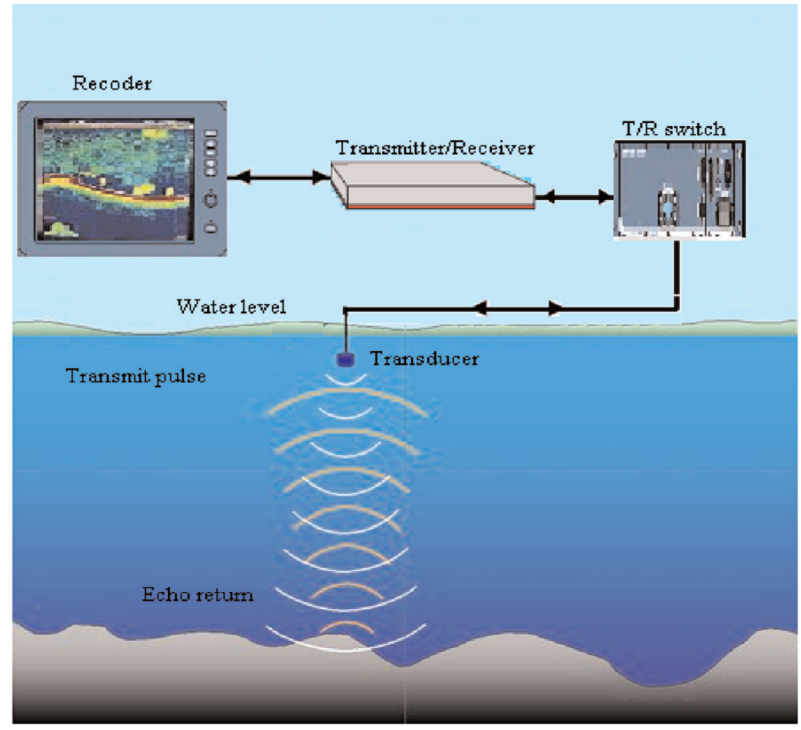

Hình 1: Phương pháp đo sâu hồi âm đơn tia
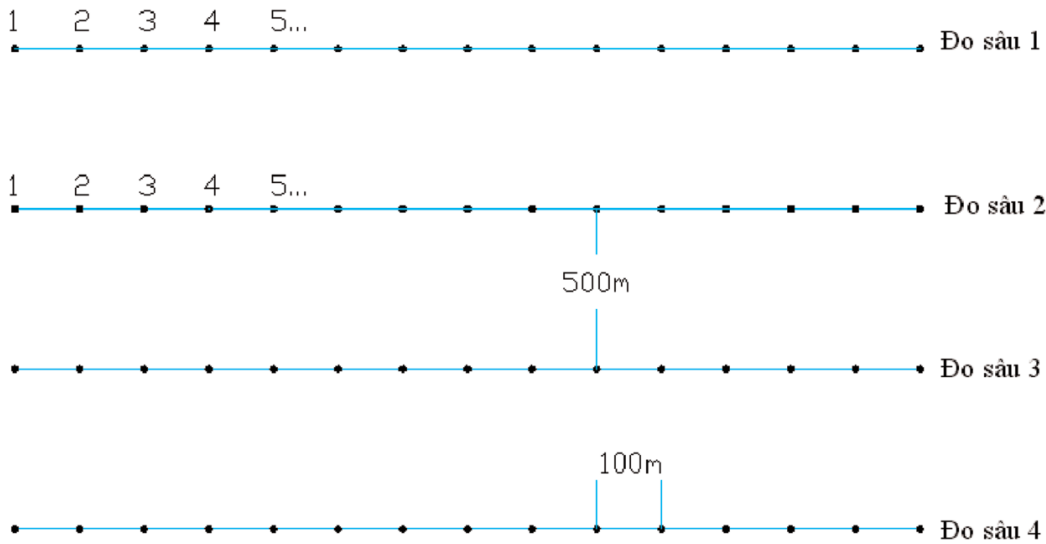

Hình 2: Sơ đồ tuyến đo sâu 
kiểm tra là $4000 \mathrm{~m}$, Tọa độ $(X, Y, D)$ của hai điểm liên tiếp trên một tuyến đo kiểm tra được xác định (Fix) với khoảng cách nhỏ hơn $20 \mathrm{~m}$. Tuyến đo kiểm tra được thiết kế như hình 3.

Khi đã có số liệu đo sâu và số liệu đo kiểm tra, chúng ta sẽ tiến hành kiểm tra độ chính xác kết quả đo sâu; quy trình kiểm tra như sau:

1 - Tìm giao điểm giữa đường đo sâu và đường kiểm tra (hình 4). Xác định góc cắt giữa đường đo sâu và đường kiểm tra, nếu góc cắt không nhỏ hơn $45^{\circ}$ và không lớn hơn $135^{\circ}$ thì thực hiện bước 2. (Xem hinh 4)

2 - Xác định tọa độ của điểm giao.

Tọa độ điểm giao $(\mathrm{P})$ được xác định bởi công thức.

$$
X_{p}=\frac{C-B}{A-D} \quad Y_{p}=\frac{A C-B D}{A-D}
$$

Trong đó:

$$
\begin{gathered}
A=\frac{Y_{D S 2}-Y_{D S 1}}{X_{D S 2}-X_{D S 1}} \\
C=\frac{Y_{K T 2}-Y_{K T 1}}{X_{K T 2}-X_{K T 1}} \\
B=\frac{X_{D S 2} \cdot Y_{D S 1}-X_{D S 1} \cdot Y_{D S 2}}{X_{D S 2}-X_{D S 1}} \\
D=\frac{X_{K T 2} \cdot Y_{K T 1}-X_{K T 1} \cdot Y_{K T 2}}{X_{K T 2}-X_{K T 1}}
\end{gathered}
$$

3. Tính độ dốc của hai điểm đo sâu liên

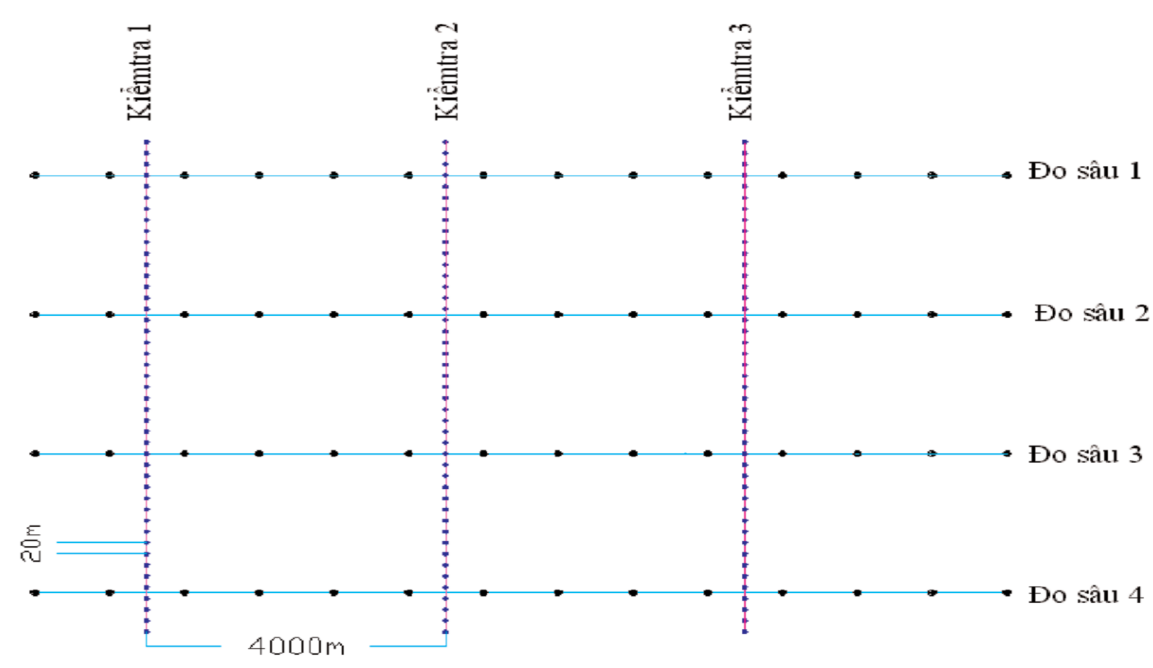

Hình 3: Sơ đồ tuyến đo kiểm tra

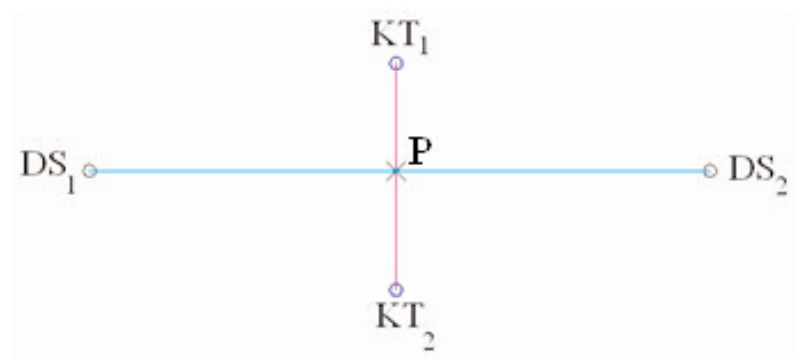

Hình 4: Giao điểm giữa đường đo sâu và đường kiểm tra 
tiếp và hai điểm kiểm tra liên tiếp có điểm giao:

$$
i_{d s}=\operatorname{arctg}\left(\frac{\Delta D_{d s}}{S_{\left(D_{D S 1} D_{D s 2}\right)}}\right) * \frac{180}{P i}
$$

với

$$
\Delta D_{d s}=D_{D S 2}-D_{D S 1}
$$

$$
i_{k t}=\operatorname{arctg}\left(\frac{\Delta D_{k t}}{S_{\left(D_{K T 1} D_{K T 2}\right)}}\right) * \frac{180}{P i}
$$

với

$$
\Delta D_{k t}=D_{K T 2}-D_{K T 1}
$$

Khi độ dốc $i_{\text {đs }}$ và $i_{k t}$ nhỏ hớn $5^{0}$ thì thực hiện bước 4 .

4 - Xác định độ sâu của điểm giao. Độ sâu tại giao điểm sẽ có 2 giá trị, đó là: độ sâu được nội suy từ 2 điểm đo sâu gần nhất trước và sau giao điểm trên từng tuyến đo sâu $\left(D_{d s}^{n o i s u y}\right)$; và độ sâu được nội suy từ 2 điểm kiếm tra gần nhất trước và sau giao điểm trên từng tuyến kiểm tra $\left(D_{k t}^{\text {noisuy }}\right)$. Hai độ sâu nội suy này là giá trị để kiêm tra độ chính xác kết quả đo sâu.

Độ sâu điểm giao $(\mathrm{P})$ được nội suy như sau:

Nội suy theo điểm đo sâu:

$$
D_{d s}^{n o i s u y}=\frac{D_{D S 1}+D_{D S 2}}{2}
$$

Nội suy theo điểm kiểm tra:

$$
D_{k t}^{n \text { oisuy }}=\frac{D_{K T 1}+D_{K T 2}}{2}
$$

5 - Xác định giá trị chênh giữa độ sâu nội suy $D_{d s}^{\text {noissyy }}$ và độ sâu suy $D_{k t}^{\text {noisuy }}$ :

$$
\Delta D=D_{d s}^{n \hat{i} s u y}-D_{k t}^{n \hat{o i s u y}}
$$

Giá trị chênh độ sâu (DD) sẽ được kiểm tra, đánh giá dựa trên các tiêu chuẩn sau:

a. Sai số trung phương của điểm đo sâu được xác định bằng công thức:

$$
m=\sqrt{\frac{\sum \Delta^{2}}{2 n}}
$$

Trong đó $\Delta(\Delta=\Delta \mathrm{D}) \Delta$ là số chênh độ sâu giữa tuyến đo sâu và tuyến đo kiểm tra tại giao điểm của 2 tuyến đo; độ sâu tại giao điểm này được nội suy từ 2 điểm đo sâu gần nhất trước và sau giao điểm trên từng tuyến đo; $n$ là số lượng giao điểm.

b. Sai số trung phương độ sâu của điểm ghi chú độ sâu sau khi đã quy đổi về hệ độ cao nhà nước điểm (được tính theo công thức (7)) không được vượt quá các hạn sai sau:

$\pm 0,30 \mathrm{~m}$ khi độ sâu đến $30 \mathrm{~m}$;

$1 \%$ độ sâu khi độ sâu trên 30m.

c. Chênh lệch độ sâu giữa điểm đo sâu và điểm kiểm tra không vượt quá 1,5 lần so với tiêu chuẩn (b) và không mang tính hệ thống.

d. Trị giá số chênh cao giới hạn của các điểm đo sâu và điểm kiểm tra không vượt quá 2 lần so với tiêu chuẩn (b) và tổng số điểm kiểm tra có số chênh từ 1,7 đến 2 lần so với quy định không được vượt quá $10 \%$ tổng số điểm kiểm tra.

Vấn đề phức tạp nhất của việc kiểm tra độ chính xác đo sâu không nằm ở việc tính toán mà nó đến từ việc tìm giao điểm giữa tuyến kiểm tra và tuyến đo. Trên thực tế một số phương pháp tìm kiếm thông thường sẽ dẫn đến độ phức tạp của thuật toán là rất lớn.

Gọi số điểm đo là $\mathrm{N}_{1}$, số điểm kiểm tra là $\mathrm{N}_{2}$, ta sẽ tính được độ phức tạp của thuật toán tìm kiếm thông thường bao gồm các công đoạn:

- Tìm kiếm hai điểm đo liền kề: $\mathrm{O}\left(\mathrm{N}_{1}{ }^{2}\right)$.

- Tìm kiếm hai điểm kiểm tra liền kề có thể giao cắt với hai điểm đo đã tìm được: $\mathrm{O}\left(\mathrm{N}_{2}{ }^{2}\right)$. 
Do hai công đoạn tìm kiếm này phụ thuộc nhau nên độ phức tạp toàn bộ thuật toán sẽ là $\mathrm{O}\left(\mathrm{N}_{1}{ }^{2} * \mathrm{~N}_{2}{ }^{2}\right)$.

\section{4. Ứng dụng mô hình TIN trong công tác kiểm tra số liệu đo sâu}

Như đã trình bày ở trên, dữ liệu đo sâu địa hình đáy biển là rất lớn từ hàng trăm nghìn đến vài triệu điểm nên khó khăn lớn nhất trong công tác kiểm tra là việc tìm giao điểm giữa đường đo sâu và đường kiểm tra. Với các thuật toán thông thường thì thời gian tìm kiếm xác định giao điểm mất vài chục phút thậm chí đến hàng giờ. Qua nghiên cứu, tìm hiểu về mô hình TIN; nhóm tác giả thấy rõ những ưu điểm của nó và đã ứng dụng mô hình TIN để tối ưu hóa việc kiểm tra độ chính xác như sau:

Từ dữ liệu đo sâu và kiểm tra $(X, Y, D)$ chúng ta xây dựng được mô hình tam giác như sau (hình 5,6 ):

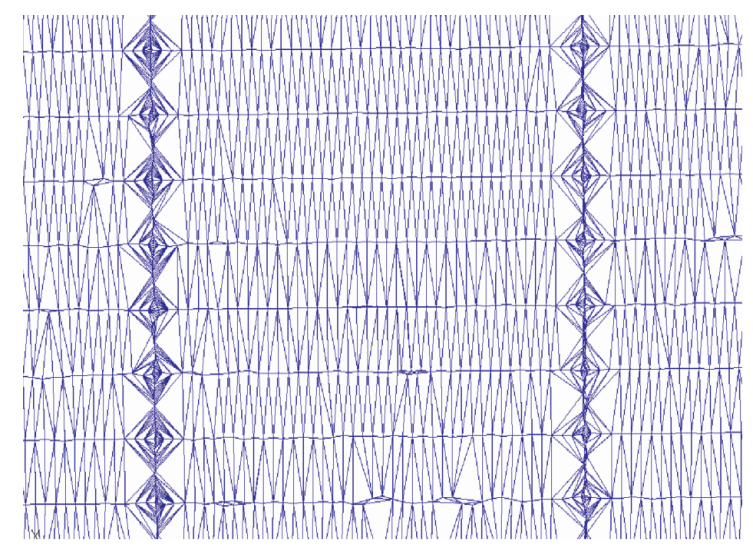

Hình 5: Mô hình tam giác hóa điểm đo sâu và điểm kiểm tra

Trên thực tế có rất nhiều phương pháp thành lập mô hình TIN với những tính năng khác nhau như phương pháp tăng dần, phương pháp quét mặt phẳng, phương pháp chia để trị... Trong đó, phương pháp tăng dần được lựa chọn vì ngoài việc xây dựng mô hình TIN, phương pháp này trang bị sẵn một cây tìm kiếm với thuộc tính topology liên kết các các tam giác trong mô hình. Điều này làm cho việc tìm kiếm điểm giao trở nên dễ dàng hơn rất nhiều.

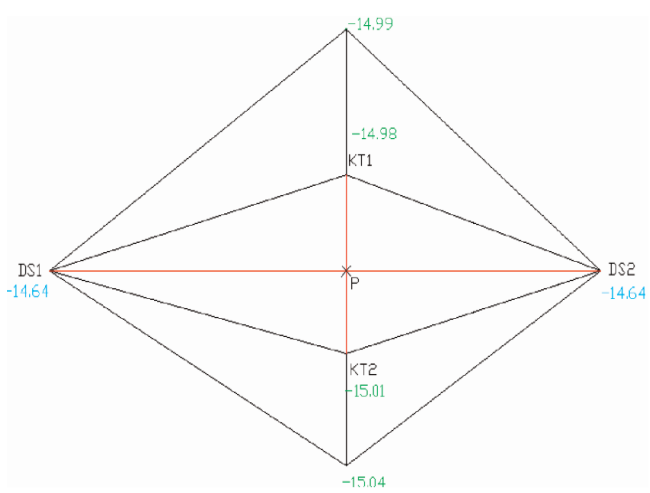

Hình 6: Mô hình tam giác hóa điểm giao cắt

Trong [1] đã nêu cụ thể phương pháp và giải thuật để xây dựng mô hình TIN theo phương pháp tăng dần. Lưu ý rằng, trong cơ sở dữ liệu điểm chúng ta phải bố trí thêm môt thuộc tính xác định điểm đo hay điểm kiểm tra.

Sử dung ngôn ngữ Visual Basic, cấu trúc điểm sẽ được mô tả như sau:

Type TPoint

Code As Byte \{Thuộc tính này xác định điểm đo hay điểm kiểm tra\}

iT As Long

$X$ As Double

$Y$ As Double

Z As Double

\section{End Type}

Cấu trúc tam giác được mô tả như sau:

Type TTriangle

TNext As Long

TCount As Byte

ID (1 To 3) As Long

iT (1 To 3) As Long

\section{End Type}

Theo đó, sau khi xây dựng xong mô hình, ta sẽ có một cơ sở dữ liệu tam giác trong đó có các thuộc tính: điểm)

Số hiệu 3 đỉnh (liên kết với cơ sở dữ liệu

Số hiệu 3 tam giác liền kề (liên kết với cơ sở dữ liệu tam giác) 


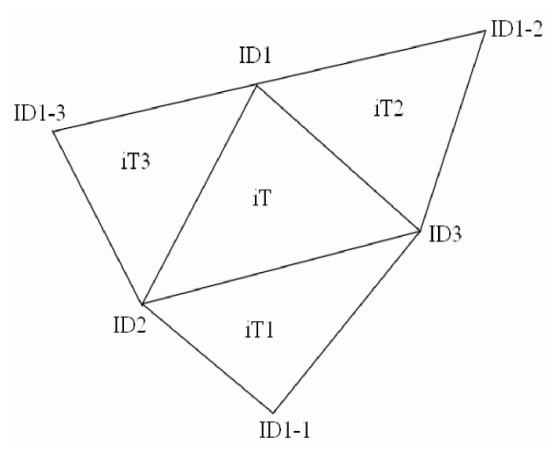

Hình 7: Mô hình cấu trúc dữ liệu tam giác

Các thuộc tính này của tam giác không những đảm bảo tính chất riêng của từng tam giác, nó còn có một ưu điểm vượt trội là khả năng liên kết topology của lưới tam giác. Khi kiểm tra một tam giác bất kỳ ta có thể dễ dàng tìm ra các tam giác liền kề nhờ 3 thuộc tính iT của tam giác đó.

Do vậy, việc tìm kiếm điểm giao khi đó chỉ đơn thuần tìm một cặp tam giác thỏa mãn điều kiện cạnh chung tạo bởi hai điểm có cùng kiểu (hoặc là điểm kiểm tra hoặc là điểm đo) và hai điểm còn lại cùng kiểu nhưng khác kiểu với kiểu của hai điểm trên cạnh chung. (Xem hinh 8)

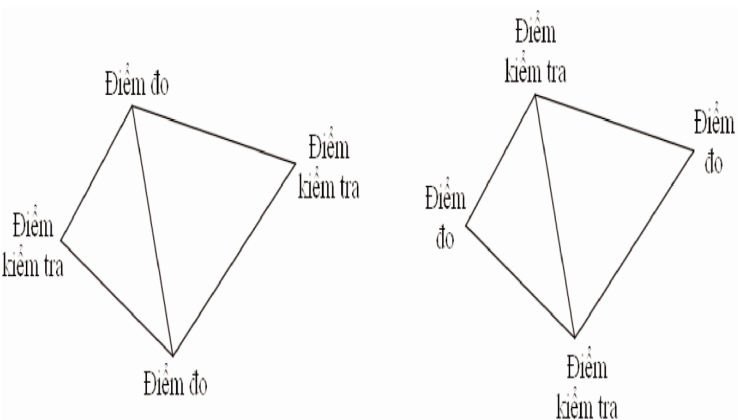

Hình 8: Cặp tam giác có cạnh chung tạo bởi hai điểm có cùng kiểu

Lưu ý rằng hai công đoạn xây dựng mô hình tam giác và công đoạn tìm kiếm điểm giao này được thực hiện độc lập nhau, tức là khi thành lập xong mô hình chúng ta mới bắt đầu tìm kiếm điểm giao. Như vậy, độ phức tạp thuật toán của toàn bộ quá trình sẽ được tính bằng tổng độ phức tạp của hai thuật toán thành phần nêu trên.

Gọi tổng số điểm đo và điểm kiểm tra là $\mathrm{N}$ ta sẽ tính được độ phức tạp của thuật toán tìm kiếm sử dụng mô hình TIN bao gồm các công đoạn:

- Lập mô hình: O(NlogN).

- Tìm kiếm tam giác thỏa mãn điều kiện có điểm giao: $\mathrm{O}(\mathrm{N} \log \mathrm{N})$.

Do hai công đoạn này độc lập nên độ phức tạp thuật toán là $\mathrm{O}(\mathrm{N} \log \mathrm{N}+\mathrm{N} \log \mathrm{N})$.

Để đánh giá tính ưu việt của thuật toán nhóm tác giả đã khảo sát tốc độ tính toán giữa phương pháp ứng dụng mô hình TIN và phương pháp duyệt thông thường, kết quả như sau: (Xem bảng 1)

\section{Nhân xét:}

So sánh độ phức tạp của hai thuật toán: tìm kiếm thông thường với độ phức tạp $\mathrm{O}\left(\mathrm{N}_{1}{ }^{2}{ }^{*} \mathrm{~N}_{2}{ }^{2}\right)$ và ứng dụng mô hình TIN với độ phức tạp $\mathrm{O}(\mathrm{N} \log \mathrm{N}+\mathrm{N} \log \mathrm{N})$ ta có thể rút ra một số nhận xét sau:

- Với tốc độ tính toán của các máy tính hiện nay thì khi khối lượng dữ liệu đầu vào nhỏ (tổng số điểm đo và kiểm tra ít hơn 2.000 điểm) thì phương pháp sử dụng mô hình TIN sẽ chưa phát huy được tính ưu việt.

- Khi dữ liệu đầu vào lớn hơn 2.000 điểm thì phương pháp này bắt đầu phát huy tốc độ tính toán và đặc biệt sẽ thể hiện rõ ưu thế khi số lượng điểm từ 5.000 điểm trở lên.

\section{Kết luận}

Mô hình TIN ngoài khả năng mô hình hóa bề mặt còn có một ưu thế rất lớn trong việc sắp xếp và tìm kiếm. Các kết quả của bài báo này chỉ ra một ứng dụng hết sức có ý nghĩa, đặc biệt trong giai đoạn hiện nay, khi công nghệ đo đạc đang được hiện đại hóa, khối lượng dữ liệu đo ngày càng lớn, đòi hỏi phải có những kỹ thuật xử lý số liệu hiện đại. 
Trao đồi - Ý kiến

\begin{tabular}{|c|c|c|}
\hline \multirow{2}{*}{ Số lượng điểm } & \multicolumn{2}{|c|}{ Thời gian tìm kiếm } \\
\cline { 2 - 3 } & $\begin{array}{c}\text { Phương pháp duyệt } \\
\text { thông thường }\end{array}$ & $\begin{array}{c}\text { Phương pháp mô } \\
\text { hinh TIN }\end{array}$ \\
\hline $\begin{array}{c}\text { Điểm đo sâu: 2.526 } \\
\text { Điểm kiểm tra: } 1.430\end{array}$ & 2.5 phút & 1.640 giây \\
\hline $\begin{array}{c}\text { Điểm đo sâu: } 9.816 \\
\text { Điểm kiểm tra: } 6.050\end{array}$ & 16.4 phút & 8.926 giây \\
\hline $\begin{array}{c}\text { Điểm đo sâu: 21.571 } \\
\text { Điểm kiểm tra: } 11.876\end{array}$ & 42.1 phút & 15.219 giây \\
\hline $\begin{array}{c}\text { Điểm đo sâu: 43.127 } \\
\text { Điểm kiểm tra: } 20.898\end{array}$ & 107.8 phút & 49.047 giây \\
\hline
\end{tabular}

Bảng 1

Bài báo này mới chỉ đề cập đến việc ứng dụng mô hình TIN trong công tác kiểm tra số liệu đo sâu bằng phương pháp đo hồi âm đơn tia, tuy nhiên, mục tiêu xa hơn là ứng dụng mô hình TIN để kiểm tra số liệu đo sâu theo phương pháp hồi âm đa tia và thành lập mô hình số địa hình đáy biển. Việc này đòi hỏi phải có những nghiên cứu tiếp theo để hoàn thiện cơ sơ khoa học và triển khai thực tiễn. $O$

\section{Tài liệu tham khảo}

[1] Nguyễn Công Sơn, Nghiên cứu một số biện pháp tối ưu hoá thành lập mô hình số địa hình, Luận văn thạc sĩ kỹ thuật, Trường Đại học Mỏ - Địa chất, Hà Nội,

\section{Summary}

2008.

[2] Đỗ Xuân Lôi, Cấu trúc dữ liệu và giải thuật, Nhà xuất bản Đại học Quốc gia, Hà Nội, 2006.

[3] Robert Sedgewick, Cẩm nang thuật toán 1-2, NXB Khoa học kỹ thuật (bản dịch), Thành phố Hồ Chí Minh, 1995.

[4] Quy định kỹ thuật thành lập bản đồ địa hình đáy biển tỷ lệ 1:50 000 (Ban hành kèm theo Quyết định số 03/2007/QĐBTNMT ngày 12 tháng 2 năm 2007 của Bộ trưởng Bộ Tài nguyên và Môi trường.

[5] Manual on Hydrography Publication M-13, 1st Edition, May 2005, Published by the international Hydrographic Bureau. $O$

\section{Applying TIN to testing resounding deep measurement data by single beam method}

MSc. Nguyen Cong Son

Vietnam Institute of Geodesy and Cartography

Dr. Tran Thuy Duong

University of Mining and Geology

Eng. Vu Hong Tap

Center for Sea Survey and Mapping

Triangular Irrigular Network (TIN) has long been known as an effective tool in setting up the terrain data model for its flexibility and good emulation of the strongly-devided terrains. Through research and practical implementation, the group of authors have found out very useful applications of TIN to modeling and organizing the exploration. This scientific article aims to introduce one of such applications. The research results of this article are a significant basis for expanding the area of application of TIN.O

Ngày nhận bài: 30/5/2013. 